\title{
Predicting Patients Found to Have Malignancy at Nipple Duct Surgery
}

\author{
Nour Alshurbasi Christopher W. J. Cartlidge Stanley R. Kohlhardt \\ Sirwan M. Hadad \\ Department of Breast and Plastic Surgery, Sheffield Teaching Hospitals, NHS Foundation Trust, Sheffield, UK
}

\section{Keywords}

Nipple discharge · Ductal carcinoma in situ .

Nipple duct surgery

\begin{abstract}
Introduction: The unexpected diagnosis of breast cancer following total duct excision is distressing for patients. Despite advances in radiology and the description of suspicious nipple discharge, pre-operative diagnosis of malignancy still evades us. The aim of this study was to review the pathological findings of total duct excision and microdochectomy with reference to pre-operative symptoms, ultrasound, or mammographic findings and identify features associated with increased likelihood of malignant disease. Methods: Data were collected retrospectively of all patients who underwent total duct excision surgery in a single centre (20112017). Pre-operative demographics, symptoms, and imaging findings were recorded and correlated with subsequent pathology. Results: 214 patients underwent total duct excision; data were available for 211 . Median age was 53 years. 175/211 (82.9\%) patients had benign pathology (duct ectasia, papilloma without atypia, fibrocystic change) on final histological examination, 21/211 (10\%) had "risk" lesions (papilloma with atypia, atypical ductal hyperplasia), and $15 / 211(7.1 \%)$ had malignancy (ductal carcinoma in situ). Of the 15 patients with malignant lesions, $6 / 15$ (40\%) had normal imaging (M1, U1). 71/211 (33.6\%) had normal imaging (M1, U1): 60/71 (84.5\%) had benign disease, 5/71 (7\%) had "risk" lesions, and 6/71 (8.5\%) had malignant lesions. 83/211 (39.3\%) patients presented with bloody discharge: $64 / 83$
\end{abstract}

(77.1\%) had benign pathology, 9/83 (10.8\%) risk, and 10/83 (12\%) malignancy. 38/211 (18\%) patients presented with non-bloody discharge: 32/38 (84.2\%) had benign disease, $4 / 38$ (10.5\%) risk, and 2/38 (5.3\%) malignant lesions. Conclusion: Neither imaging nor presenting symptoms correlate with the likelihood of malignant disease being present at final pathology. Even with advances in pre-operative diagnosis, total duct excision remains an essential diagnostic and therapeutic procedure.

(c) 2019 S. Karger AG, Basel

\section{Introduction}

Total duct excision is a frequently performed procedure, most usually undertaken for the common symptom of nipple discharge [1]. Nipple discharge is the third most common cause of referral to breast services [2], subsidiary only to the finding of a lump or the presence of pain [3]. It has been estimated that it may account for $2-5 \%$ of all medical consultations undertaken by women and may be the presenting symptom in 5-12\% of breast cancers [4]. The finding of occult malignancy in the pathological specimen taken at total duct excision surgery is an unusual occurrence and is alarming for both patient and surgeon [5]. If there were a reliable way to determine the likelihood of malignant disease being found at total duct

N.A. and C.W.J.C. are joint first authors. S.R.K. and S.M.H. are the senior authors. 
excision, it could reduce unnecessary anxiety and delay associated with this unexpected event.

Previous work looking at predictors of malignancy in patients with nipple discharge has suggested that older age and the presence of a suspicious lump are most likely to be associated with malignant disease [6]. However, this did not specifically look at patients who underwent duct excision as a primary procedure, and we would expect most malignancy with an associated suspicious lump to be picked up on standard triple assessment and treated by wide local excision or mastectomy in the first instance. Other studies of patients with nipple discharge have shown carcinoma in 3\% (up to $9 \%$ of those who underwent biopsy), but again these data also include those presenting concurrently with a lump [7]. A study of patients with pathological nipple discharge (defined as unilateral, spontaneous, bloody, or clear) but normal imaging showed only $1 / 48(2.1 \%)$ cases with a small in situ malignancy and suggested that observation may be safe in these patients [8].

We do not yet know whether the assessment of symptoms (by type of discharge and presence of blood) can be combined with the use of modern imaging (digital mammography and high-definition ultrasound) to adequately risk-stratify patients prior to total duct excision, or even predict those who will go on to have malignancy found at final histopathology. Recent trials have focused on the use of ductoscopy [9] and MRI [10], but these are either expensive and time consuming (MRI) or not commonly available (ductoscopy).

The aims of this study are to ascertain the pathological outcomes of total duct excision in a large single-centre series to determine whether the procedure is necessary for the diagnosis of malignancy in patients who present solely with nipple symptoms and to establish predictors of malignant pathology using presenting symptoms or imaging findings.

\section{Materials and Methods}

All patients who had undergone a procedure coded as total duct excision or microdochectomy between January 2011 and April 2017 at a single teaching hospital were identified. A retrospective review of the notes was conducted, and data were collected for age, presenting symptoms, radiology findings, and final histopathological evaluation. Patients already known to have breast cancer were excluded. Appropriate ethical approval was obtained from the institution's research department.

New referrals with nipple discharge were routinely examined in a new patient clinic by an experienced breast clinician or surgeon and then underwent imaging. All of the patients in this study were assessed based on the new patient clinic protocol, and there were no patients who underwent surgery directly following screening mammography. According to the commonly agreed unit protocol, patients less than 40 years had ultrasound, performed using high-frequency transducers to assess the sub-areo- lar tissue. Patients over 40 years also underwent diagnostic bilateral mammography using standard craniocaudal and mediolateral oblique views. Mammograms were performed when the period of time since the last mammogram was over 1 year, and where previous mammograms existed the contemporary images were compared with the historic. Imaging assessment was according to the BI-RADS classification and scored as one of: $\mathrm{U} 1 / \mathrm{M} 1=$ normal, U2/M2 = benign, U3/M3 = probably benign, U4/M4 = suspicious, and U5/M5 = highly suggestive of malignancy. All mammograms were reported by and ultrasound scans were performed and reported by experienced specialist breast radiologists with at least 5 years of experience of the techniques. No patient who underwent total duct excision had imaging findings of $U 4 / 5$ or $\mathrm{M} 4 / 5$, and this seems appropriate as excision biopsy or wide local excision (depending upon biopsy results) is more often required in these cases.

Total duct excision was undertaken using a radial areolar incision, as subsequently described [11]; all nipple ducts were disconnected from the underside of the nipple, and a cone of tissue excised and sent to histopathology. The nipple side of the excised tissue was marked using a silk suture to aid the histopathologist with orientation and allow assessment of the adequacy of deep margins should malignant or atypical disease be found.

Histopathology reports were examined for all patients who had undergone total duct excision and the final pathological determination recorded. Ductal carcinoma in situ (DCIS) or invasive carcinoma were defined as malignant lesions; duct ectasia, fibrocystic change, and papilloma without atypia were defined as benign lesions; atypical ductal or lobular hyperplasia or papilloma with atypia were defined as risk marker lesions.

Statistical analysis was performed using Microsoft Excel and Student's $t$ test for continuous variables and a $\chi^{2}$ test for discrete variables. Significance was determined by a $p \leq 0.05$.

\section{Results}

\section{Demographics}

In total, 214 patients underwent total duct excision; data were available for 211 . All were female with a median age of 53 years (range 19-87 years). Of the 211 patients, $175(82.9 \%)$ had a final pathology that was benign: 93/175 (53.1\%) duct ectasia; 64/175 (36.6\%) papilloma; $8 / 175$ (4.6\%) fibrocystic change; $3 / 175$ (1.7\%) inflammatory changes; $2 / 175$ (1.1\%) nipple adenoma, $2 / 175(1.1 \%)$ periductal mastitis; $1 / 175(0.6 \%)$ normal ducts only; $1 / 175(0.6 \%)$ usual hyperplasia; and $1 / 175(0.6 \%)$ simple cyst. Twenty-one of the 211 (10.0\%) patients had pathology demonstrating lesions associated with increased risk: 7/21 (33.3\%) multiple papillomas; 6/21 (28.6\%) papilloma with atypical ductal hyperplasia; 4/21 (19.0\%) atypical ductal hyperplasia alone; $2 / 21$ (9.5\%) papilloma with radial scar; $1 / 21$ (4.8\%) papilloma with lobular carcinoma in situ; and 1/21 (4.8\%) fibrocystic change with radial scar. Fifteen (7.1\%) patients had malignant lesions: 4/15 (26.7\%) high nuclear grade DCIS; $7 / 15$ (46.7\%) intermediate nuclear grade DCIS; $1 / 15$ (6.7\%) low nuclear grade DCIS, and 3/15 (20\%) DCIS of unknown nuclear grade. 
Fig. 1. Presenting symptom by number of patients with each.

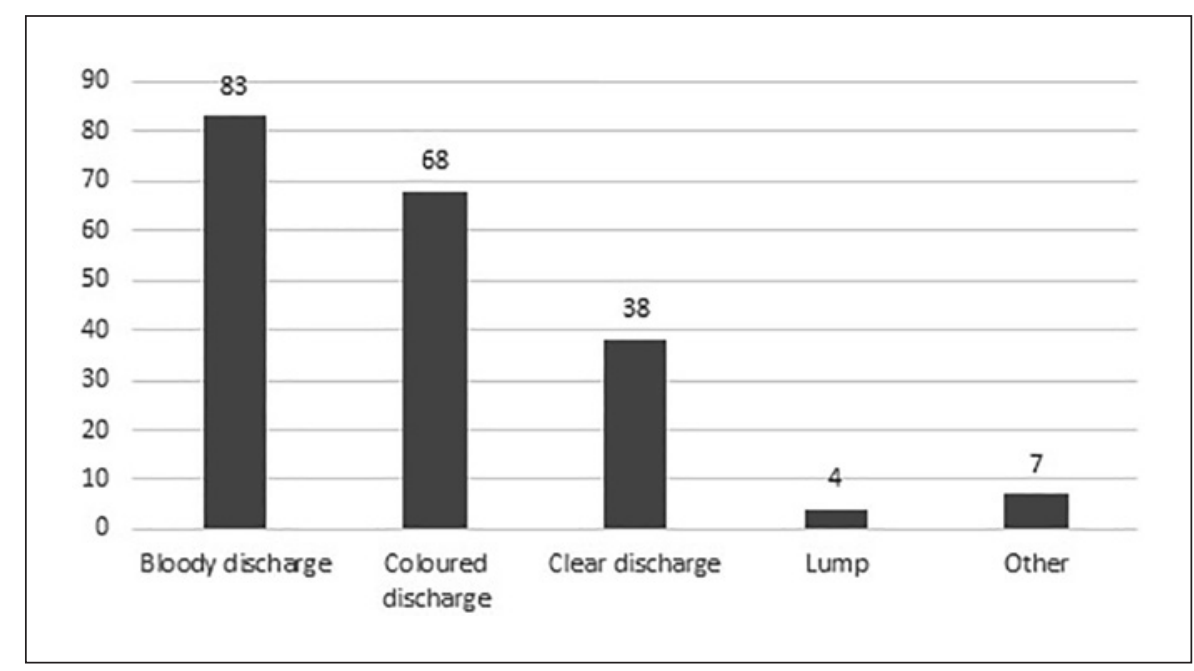

\section{Presentation}

Figure 1 shows the most common presentation was bloody discharge $(83 / 211 ; 39.3 \%$ patients), followed by "coloured" discharge $(68 / 211 ; 32.2 \%)$, then clear or serous discharge $(38 / 211 ; 18.0 \%)$, then a lump $(4 / 211$; $1.9 \%)$, and others including recurrent mastitis $(1 / 211$; $0.5 \%)$, nipple fistula $(2 / 211 ; 0.9 \%)$, nipple inversion $(2 / 211 ; 0.9 \%)$, and nipple distortion $(2 / 211 ; 0.9 \%)$. There was no record of the presenting complaint in 11/211 (5.2\%) patients.

\section{Relationship of Presentation to Imaging Findings and} Final Pathology

Of the patients presenting with bloody discharge, 40/83 (48.2\%) had normal imaging (U1 and M1 where mammogram was performed, i.e., $>40$ years old). Thirty of $83(36.1 \%)$ had imaging showing benign changes (U2 or M2), and $10 / 83(12.0 \%)$ had indeterminate imaging (U3 or M3). For the rest of the patients $(3 / 83 ; 3.6 \%)$, there were no imaging findings recorded - they may have had recent normal breast screening imaging and reassuring examination findings. On final histopathology of the patients presenting with bloody discharge, 64/83 (77.1\%) had benign findings, $10 / 83(12.0 \%)$ patients had increased risk lesions, and 9/83 (10.8\%) patients had malignant lesions.

Figure 2 shows imaging findings for each category of presenting complaint. Of the patients with coloured discharge: 35/68 (51.5\%) had normal imaging (U1 +/- M1), 27/68 (39.7\%) had benign imaging (U2 or M2), 5/68 (7.4\%) had indeterminate imaging, and 2/68 (2.9\%) had no imaging. On final histopathology, 60/68 (88.2\%) patients had benign findings, $6 / 68(8.8 \%)$ had increased risk lesions, and 2/68 (2.9\%) had malignancy.

Of patients with clear discharge, 20/38 (52.6\%) had normal imaging (U1 +/- M1), 13/38 (34.2\%) had benign findings, and $5 / 38(7.4 \%)$ had indeterminate findings on imaging. On final histopathology, 32/38 (84.2\%) had benign findings, $4 / 38$ (10.5\%) had increased risk lesions, and $2 / 38(5.3 \%)$ had malignancy.

Where the patient presented with a lump, all $4 / 4$ (100\%) had indeterminate imaging (U3 or M3). These patients all underwent triple assessment which gave a biopsy result of indeterminate lesion (B3). Due to a low suspicion of malignancy, total duct excision was performed, and on final histopathology, $3 / 4$ (75\%) had benign findings, and 1/4 (25\%) had an increased risk lesion (papilloma with atypia). More recently, these lesions might have been treated by large-volume vacuum-assisted biopsy.

For patients with other presentations or where there was no record of the presenting complaint, 7/18 (38.9\%) had normal imaging, 5/18 (27.8\%) had benign imaging findings, $5 / 18(27.8 \%)$ had indeterminate imaging, and $1 / 18(5.6 \%)$ had no record of imaging findings. On histopathology following total duct excision, 16/18 (88.9\%) patients had benign findings, $1 / 18(5.6 \%)$ had increased risk lesion, and 1/18 (5.6\%) had malignancy.

Figure 3 shows the final pathology for each of the categories of presenting complaint. When comparing the imaging outcomes for the 3 most common presentations (bloody discharge, coloured discharge, and clear discharge), there was no significant difference in the distribution of imaging outcomes for each symptom ( $p=$ 0.595). Similarly, when comparing the final histopathology results across all the categories of presenting symptoms, there was no significant difference in the proportion of each category that had benign, risk, or malignant pathology $(p=0.613)$.

\section{Relationship of Imaging Findings to Final Pathology}

Across all presentations, 102/211 (48.3\%) patients had normal imaging, 75/211 (35.5\%) had benign imaging findings, and 29/211 (13.7\%) had indeterminate imaging 


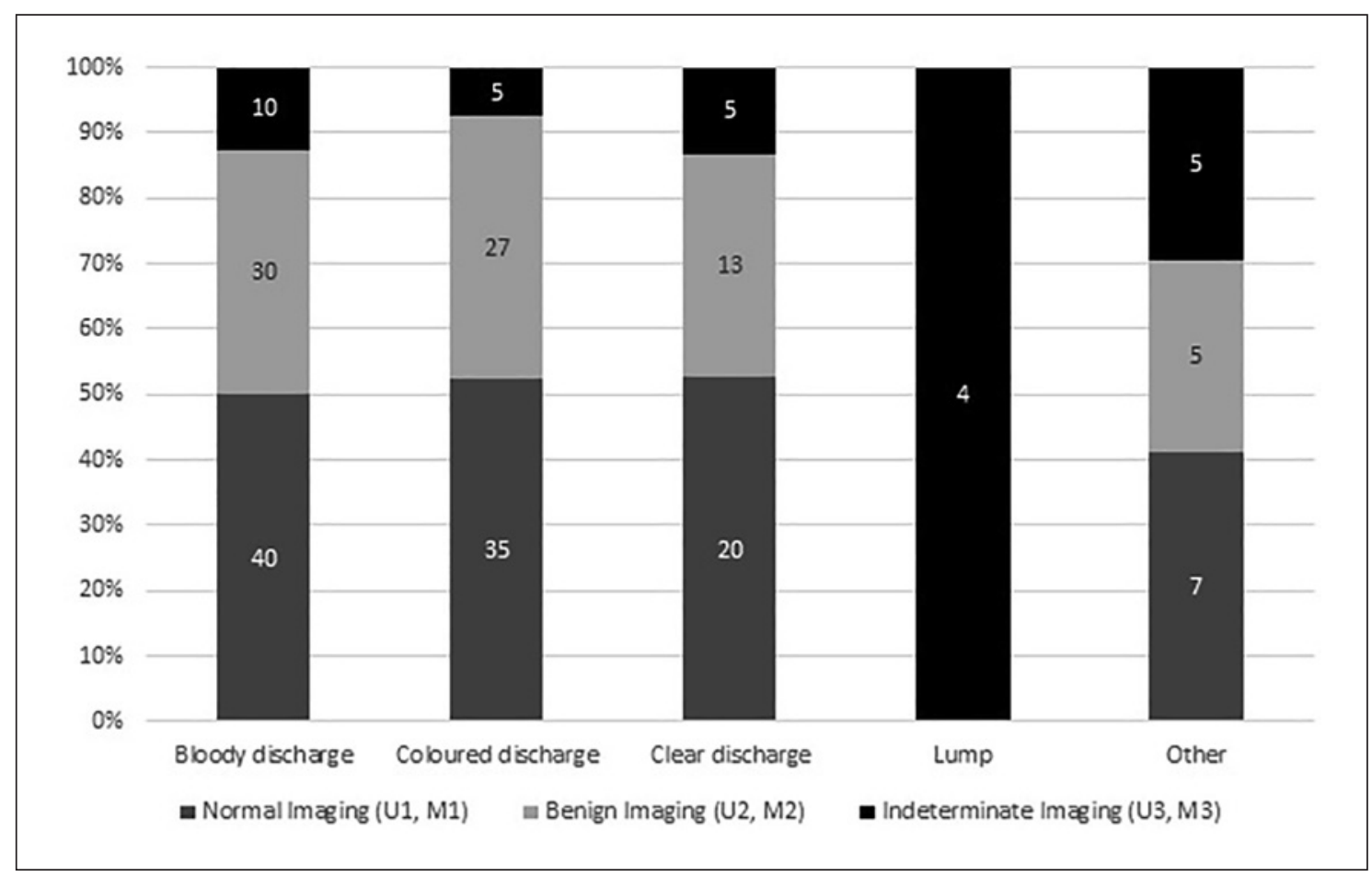

Fig. 2. Imaging findings by presenting symptom (patients without imaging excluded); numbers indicate the patients in each category.

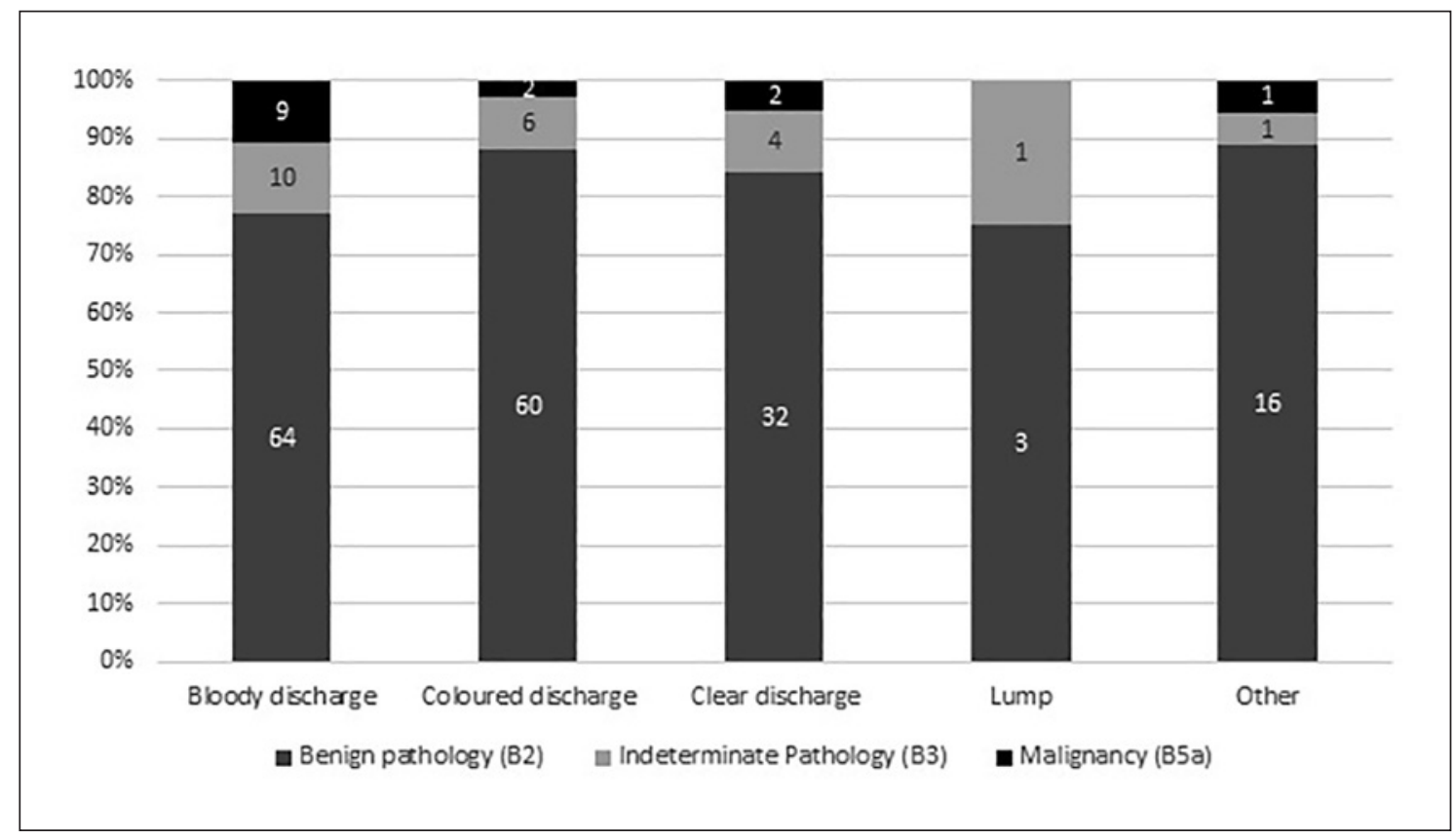

Fig. 3. Final histopathology findings by presenting symptom; numbers indicate the patients in each category.

(U3 or M3). Five of 211 (2.4\%) patients had no imaging recorded.

Of those with normal imaging, 90/102 (88.2\%) had benign findings only on final histopathology, 6/102 (5.9\%) had increased risk lesions, and 6/102 (5.9\%) had malignancy. Of those with benign imaging, $57 / 75(76 \%)$ had benign final histopathology, 10/75 (13.3\%) had increased risk lesions, and $8 / 75$ (10.7\%) had malignancy. When the 


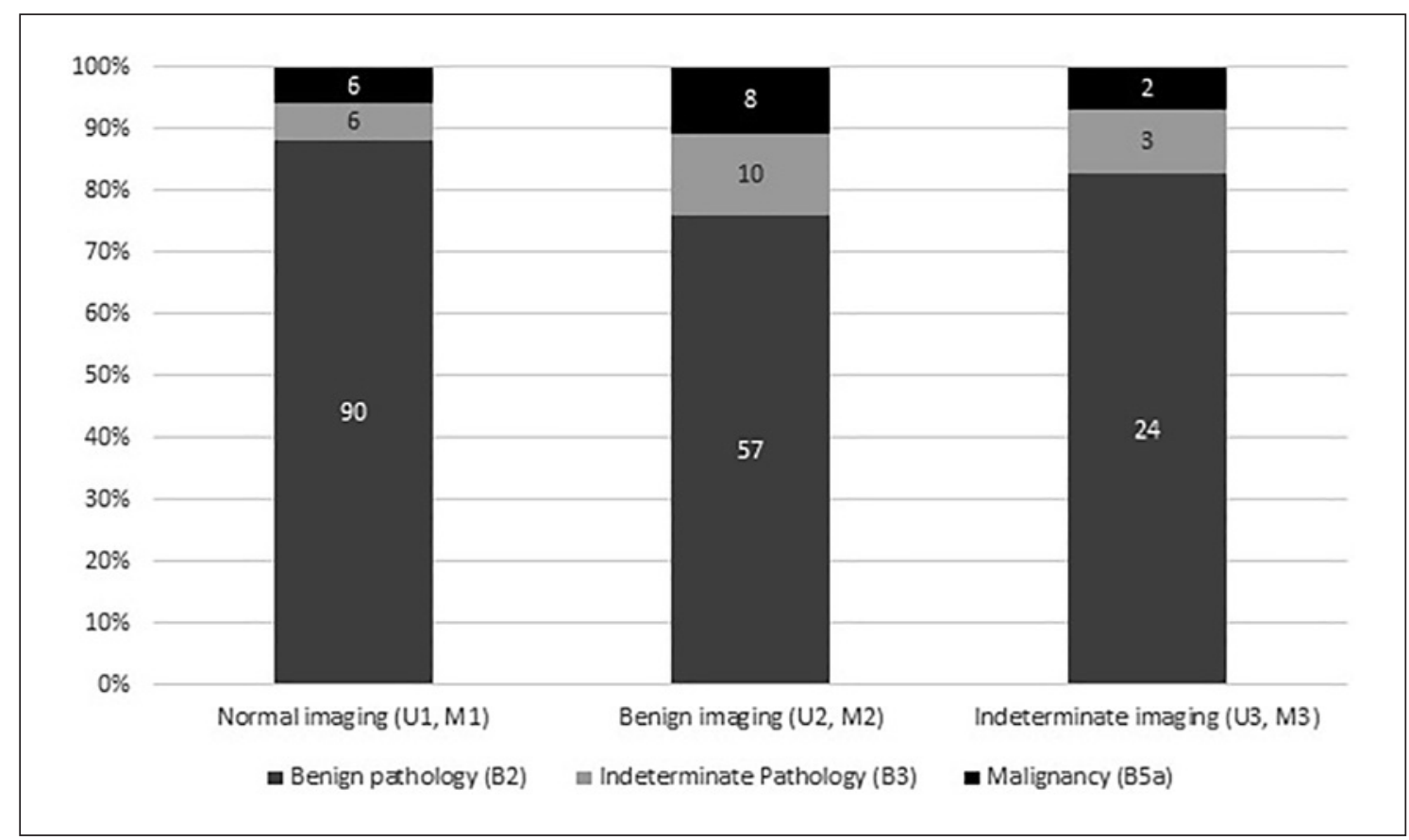

Fig. 4. Final histopathology outcome by imaging findings; numbers indicate the patients in each category.

patient had indeterminate imaging (U3 or M3) then on final histopathology: 24/29 (82.8\%) had benign findings, $3 / 29(10.3 \%)$ had increased risk lesions, and 2/29 (6.9\%) had malignant lesions.

Comparing final histopathology (Fig. 4) across each of the categories of imaging findings (normal, benign, and indeterminate), there was no significant difference in the proportion of benign, risk, or malignant disease in each patient group $(p=0.316)$.

There were a small number of patients in whom imaging was discordant. Twelve patients given an M1 score were subsequently scored as U3. All of these patients had normal or benign tissue on final pathology, and as the most common U3 imaging finding was a likely papilloma, the reason for the discrepancy was thought to be the small size of the lesion. Two patients had an M3 but a U1 or U2 score, and this was due to the presence of calcification or multiple papillomas on the mammogram. Again, the final pathology in these cases was always normal or benign.

\section{Comparison of Patients with Malignant Disease and}

Those with Benign Final Pathology

Table 1 compares patients found to have malignant disease with those found to have benign disease in terms of age and findings on imaging. Of patients with malignant disease, $6.7 \%$ had indeterminate (U3) ultrasound imaging compared to $12.3 \%$ of those with benign disease. Figure 5 shows the distribution of age ranges as a box plot
Table 1. Findings in patients with malignant disease compared to benign histopathology

\begin{tabular}{lll}
\hline & Malignant & Benign \\
\hline $\begin{array}{l}\text { Median age, years } \\
\text { Patients, } n\end{array}$ & 66 & 51 \\
U1 imaging & $8(53.3 \%)$ & $83(53.5 \%)$ \\
U2 imaging & $6(40 \%)$ & $53(34.2 \%)$ \\
U3 imaging & $1(6.7 \%)$ & $19(12.3 \%)$ \\
\hline
\end{tabular}

(minima, maxima, interquartile range, median [line], and mean $[\mathrm{X}]$, outlier beyond lower limit for malignant) in each of the 3 final pathology groups. Patients with malignant disease tended to be older although there was no significant difference.

\section{Discussion/Conclusion}

The most common presenting complaint prior to total duct excision was nipple discharge, with bloody discharge being the most prevalent when compared to other coloured or clear discharge. Every symptomatic group had a small number of cases diagnosed with malignancy on final pathology, except for those presenting with a lump, which probably represents more accurate pre-operative diagnosis due to the ability to undertake core biopsy in 
Fig. 5. Age of the patients with benign, intermediate, or malignant final pathology.

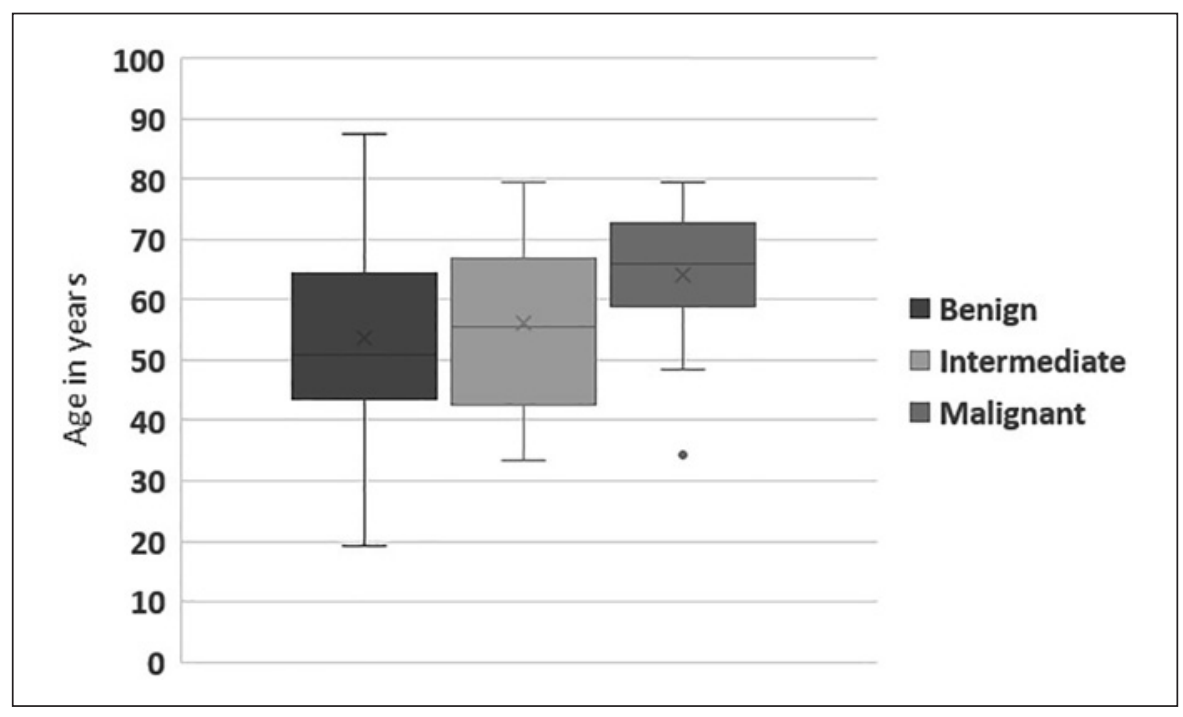

these patients. There was no clear way to risk stratify patients based on presenting symptoms. Similarly, imaging findings could not accurately herald patients who would go on to be found to have malignant disease on histopathology, with no significant difference in the proportion of those with normal, benign, or indeterminate imaging going on to have malignant disease.

In this series, $7.1 \%$ of patients were found to have malignancy. A previously published series [12] showed a similar overall figure (5\%) of patients who were found to have malignancy, although the majority of these patients (6 out of 7) had abnormal mammogram or ultrasound. In our study, even with entirely normal mammogram and ultrasound scan, $5.9 \%$ of patients were subsequently found to have malignancy. In another major series, 5.7\% of patients were found to have malignancy [2], but they found a clearer association with those patients presenting with bloody discharge who had a rate of malignancy of $10.2 \%$. Interestingly, they had 4 cases of invasive malignancy out of 7 in total.

A previous study of 86 patients undergoing surgery for nipple discharge found only 1 with DCIS (1.2\%) and was taken to illustrate the rarity of such an occurrence [5]. When the pathology of 97 patients who underwent microdochectomy for single-duct discharge was examined, $8(8.2 \%)$ patients were found to have DCIS [13]. However, $6 / 8$ of these patients had pre-operative mammograms that were suspicious of malignancy. Thus, the rate of unexpected malignancy was $2 / 97$ (2.1\%).

In our cohort, we could not identify a subgroup of nipple discharge that was more likely to be malignant dependent on colour. Statistically, having blood-stained, coloured, or clear discharge, all had the same incidence to harbour cancer in final pathology $(p=0.595)$. Clinically, this is important, as clinicians tend to assume that the dis- charge is more pathological or signifies higher risk when it is blood stained rather than coloured.

Imaging using mammography with or without ultrasound scanning failed to identify a higher risk group ( $p=$ $0.316)$. This poor correlation has been noted in previous studies [14]: they showed an overall malignancy rate of $15 \%$ but noted that even MRI scanning could only provide a positive predictive value of $56 \%$ and negative predictive value of $87 \%$ in cases of "suspicious" nipple discharge. Our findings are at odds with others [8] who have reported very low rates of malignant disease in patients with normal imaging.

Work comparing the modalities showed that MRI was superior to galactography in detecting malignancy [15] in patients with nipple discharge with normal mammograms. MRI sensitivity in this context was $95.7 \%$, and specificity was $69.7 \%$. However, the cost and impact on radiology reporting workload of breast MRI make it less appealing as a diagnostic tool, and additionally a longer delay to surgical diagnosis as the gold standard would not be well tolerated.

One limitation of this study in assessing the rates of malignant disease is that obviously cancerous lesions (in particular those with a breast mass) have already been sifted out by the normal process of triple assessment. This may explain why no invasive disease was found in this series. However, it also emphasises the importance of being able to predict when such patients who have otherwise been "cleared" by traditional methods may still harbour serious disease. Despite this, as mentioned above, the overall rate of malignancy in this study was similar to or slightly higher than that seen in other series. There is no obvious explanation for this incongruity, and it may simply reflect regional or temporal differences in the rates of pre-invasive disease. 
A further factor is that nipple discharge is a very common symptom, and so we are very likely to only be seeing a self-selected group of patients who submit to surgery. It is possible that other symptoms that are not so easily recorded in the assessment process may relate to the likelihood of finding malignancy. We were unable to obtain data on the duration of symptoms for patients who presented, and this series is limited to patients who underwent surgery, and so we might presume that their symptoms were significantly troublesome in some way.

There is no clearly identifiable factor in clinical presentation or radiological investigation findings to predict patients who will be shown to have malignant disease at final histopathology. Total duct excision remains the gold standard for the simultaneous diagnosis and treatment of disorders of nipple discharge and is necessary for the diagnosis of malignancy in a subset of patients. At this stage, we cannot reliably exclude patients from the procedure based on any specific symptomatology or imaging finding. We would recommend that total duct excision is offered to any patient with persistent nipple discharge in the absence of another diagnosis on the basis that a proportion of these patients will be found to have malignant disease.

\section{Statement of Ethics}

This research complies with the guidelines for human studies and was conducted ethically in accordance with the World Medical Association Declaration of Helsinki. As the study involved retrospective data collection, subjects are not individually identifiable, and there was no alteration to standard treatment, individual subject consent was not required.

\section{Disclosure Statement}

The authors have no conflicts of interest to declare.

\section{Funding Sources}

Funding sources were not available.

\section{Author Contributions}

N.A. and C.W.J.C. contributed equally to data collection, analysis, and drafting of the manuscript. S.R.K. and S.M.H. contributed to supervising the research, guiding, and revising the manuscript.

\section{References}

1 Mansel RE, Webster D, Sweetl HM. Hughes, Mansel \& Webster's benign disorders and diseases of the breast. London: Saunders Ltd; 2009.

2 Foulkes RE, Heard G, Boyce T, Skyrme R Holland PA, Gateley CA. Duct excision is still necessary to rule out breast cancer in patients presenting with spontaneous bloodstained nipple discharge. Int J Breast Cancer. 2011; 2011:495315.

3 Dixon JM. ABC of breast diseases. 4th ed. Chichester/Hoboken: Wiley-Blackwell; 2012.

4 Patel BK, Falcon S, Drukteinis J. Management of nipple discharge and the associated imaging findings. Am J Med. 2015 Apr;128(4): 353-60.

5 Richards T, Hunt A, Courtney S, Umeh H. Nipple discharge: A sign of breast cancer? Ann R Coll Surg Engl. 2007 Mar;89(2):124-6.

6 Jin L, Zhu L, Li S, Zeng Y, Haixiong L, Su F, et al. Predictors of malignancy for female patients with suspicious nipple discharge: A retrospective study. Anticancer Res. 2017 Aug; 37(8):4655-8.
7 Gray RJ, Pockaj BA, Karstaedt PJ. Navigating murky waters: a modern treatment algorithm for nipple discharge. Am J Surg. 2007 Dec; 194(6):850-4.

8 Li GZ, Wong SM, Lester S, Nakhlis F. Evaluating the risk of underlying malignancy in patients with pathologic nipple discharge. Breast J. 2018 Jul;24(4):624-7.

9 Gui G, Agusti A, Twelves D, Tang S, Kabir M, Montgomery C, et al. INTEND II randomized clinical trial of intraoperative duct endoscopy in pathological nipple discharge. Br J Surg. 2018 Nov;105(12):1583-90.

10 Bahl M, Gadd MA, Lehman CD. JOURNAL CLUB: Diagnostic utility of MRI after negative or inconclusive mammography for the evaluation of pathologic nipple discharge. AJR Am J Roentgenol. 2017 Dec;209(6): 1404-10.
11 Baker BG, Mathen V, Harvey JR. Total duct excision through a linear radial areola incision. Breast J. 2018 May;24(3):446-7.

12 Sabel MS, Helvie MA, Breslin T, Curry A, Diehl KM, Cimmino VM, et al. Is duct excision still necessary for all cases of suspicious nipple discharge? Breast J. 2012 Mar-Apr; 18(2):157-62.

13 Locker AP, Galea MH, Ellis IO, Holliday HW, Elston CW, Blamey RW. Microdochectomy for single-duct discharge from the nipple. $\mathrm{Br}$ J Surg. 1988 Jul;75(7):700-1.

14 Morrogh M, Morris EA, Liberman L, Borgen PI, King TA. The predictive value of ductography and magnetic resonance imaging in the management of nipple discharge. Ann Surg Oncol. 2007 Dec;14(12):3369-77.

15 Lubina N, Schedelbeck U, Roth A, Weng AM, Geissinger E, Hönig A, et al. 3.0 Tesla breast magnetic resonance imaging in patients with nipple discharge when mammography and ultrasound fail. Eur Radiol. 2015 May;25(5): 1285-93. 